RESEARCH ARTICLE

\title{
Mandrel degradation model of combined fast and slow processes
}

\author{
Yu Zhu ${ }^{1}$, Zheng Liu ${ }^{1}$, Famin $\mathrm{Yu}^{1}$, Qiang $\mathrm{Chen}^{2}$, Wei Feng ${ }^{1}$, Zhanwen Zhang ${ }^{2}$, and Zhigang Wang ${ }^{1}$ \\ ${ }^{1}$ Institute of Atomic and Molecular Physics, Jilin University, Changchun 130012, China \\ ${ }^{2}$ Laser Fusion Research Center, China Academy of Engineering Physics, Mianyang 621900, China \\ (Received 11 September 2020; revised 28 October 2020; accepted 24 November 2020)
}

\begin{abstract}
In this paper, we report the study of degradation for a kind of ideal mandrel material called poly- $\alpha$-methylstyrene based on theoretical and experimental methods. First-principles calculations reveal two types of process: depolymerization and hydrogen-transfer-induced chain scission. The energy barrier for the former $(0.68-0.82 \mathrm{eV})$ is smaller than that for most of the latter $(1.39-4.23 \mathrm{eV})$. More importantly, reaction rates suggest that the former is fast whereas the latter is mostly slow, which can result in a difference of 5-31 orders of magnitude at $550 \mathrm{~K}$. Furthermore, a thermogravimetric experiment shows that the activation energy of $2.53 \mathrm{eV}$ for degradation is between those of fast and slow processes, corresponding to the theoretical average value of multiple reaction paths. Thus, a mandrel degradation model combining fast and slow processes is established at the atomic level. Our work provides a direction for research into the key technology of target fabrication in inertial confinement fusion.
\end{abstract}

Keywords: atomic level; degradation; first principles; mandrel material; thermogravimetric experiment

\section{Introduction}

As an important method for fabricating inertial confinement fusion (ICF) targets, the degradable mandrel technique is the basis of preparing glow discharge polymer (GDP) microspheres ${ }^{[1-4]}$. It can be divided into three steps ${ }^{[2,3]}$ : first, hollow polymer microspheres are prepared as mandrels; then, plasma vapour deposition technology is used to prepare a coating with higher thermal stability on the surface; and, finally, the coated mandrels are degraded leaving hollow microspheres. In these steps, the preparation of hollow microspheres is directly related to the degradation properties of polymers. The technique requires that coatings are thermostable at the degradation temperature of polymers, and degradation products do not affect the formation and surface finish of microspheres. To meet these requirements, poly$\alpha$-methylstyrene (PAMS) has become an ideal choice for mandrel materials (MMs), because of its excellent thermal degradation properties and good balling performance ${ }^{[5,6]}$.

Correspondence to: Z. Wang, No. 2699, Qianjin Road, Changchun 130012, China; Z. Zhang, No. 64, Mianshan Road, Mianyang 621900, China. Email: wangzg@jlu.edu.cn (Z. Wang); bjzzw1973@163.com (Z. Zhang)
However, it still presents two key problems in preparing target pellets, namely how to reduce the thermal degradation temperature and avoid degradation residues ${ }^{[2,7]}$. These lead to the urgent need for a clear understanding of the reaction mechanism of MM-PAMS degradation.

In the past, there have been numerous reports on the thermal degradation of MM-PAMS ${ }^{[8-17]}$. Researchers have generally believed that degradation was caused by, first, generating free radicals through random chain scission and, then, depolymerizing to generate monomers ${ }^{[9,10]}$. However, it should be noted that these two processes are not recognized at the atomic level, resulting in an order of magnitude difference in reaction rate between them that remains unclear. More importantly, a precise description of these processes based on the properties of geometric and electronic structures is necessary for controlling the degradation process and avoiding degradation residues. It is undeniable that experiments can obtain some reliable results, but calculations and simulations of quantum mechanics have also become an indispensable tool in understanding the reaction process and mechanism of microscopic matter, and have shown sufficient accuracy in degradation or general chemical reactions to attract widespread attention ${ }^{[16,18-21]}$. 
In this research, we conducted the theoretical and experimental study of MM-PAMS degradation. Surprisingly, we found that it has two processes, fast and slow, with vastly different reaction rates. Our work highlights the decisive role of the slow process in the degradation of MMs, and provide a direction for effective regulation.

\section{Methods}

Both theoretical and experimental methods were adopted to study the degradation process and mechanism of MMPAMS. Based on detailed analysis of these two aspects, we built a degradation model at the atomic level.

For theoretical calculations, we used the high-precision method of density functional theory (DFT) to study the potential energy surfaces (PESs) of various possible degradation paths. Specifically, all extreme-point structures of PESs were optimized by using the exchange-correlation functional of generalized gradient approximation (GGA) with empirical dispersion correction B3LYP-D3 ${ }^{[22,23]}$, in conjunction with $6-31+\mathrm{G}(\mathrm{d}, \mathrm{p})$ basis sets. The frequency calculations were also performed at the same level to determine the stability of structures for extreme points, that is, the numbers of imaginary frequencies were used to confirm that the optimized structure was a stable point or transition state (TS). Meanwhile, the zero-point energy (ZPE) was also determined, and the intrinsic reaction coordinate (IRC) ${ }^{[24,25]}$ was calculated to ensure the reliability of the reaction processes. These calculations were all performed using the Gaussian 09 software package ${ }^{[26]}$. In addition, we corrected spin contamination in the low-spin state with an approximate spin-projection method ${ }^{[27,28]}$. As for dynamic simulation, the empirical dispersion-corrected self-consistent charge density functional tight-binding (SCC-DFTB-D) ${ }^{[29,30]}$ method was used, which is $10^{3}$ to $10^{4}$ times faster than DFT on the basis of meeting the maximum accuracy requirements, so it was suitable for our system. The dynamic simulations were implemented in the DFTB $+19.1 \operatorname{program}^{[31]}$.

To complete the experimental observation, we used the thermogravimetric (TG) method to measure the TG curve of MM-PAMS. The sample used in the experiment was a synthetic product with average molecular weight of $1.14 \times 10^{6}$ and polydispersity index of 1.55 . The experimental setup used a TG analyser. The selected temperature range was from room temperature to $600^{\circ} \mathrm{C}$, and the heating rate was $5^{\circ} \mathrm{C} / \mathrm{min}$. The carrier gas was Ar with flow rate of $2.5 \mathrm{~L} / \mathrm{h}$. The sample dosage was $20-30 \mathrm{mg}$.

\section{Results and discussion}

Considering the reliability and resource requirements of quantum calculations, two typical chain-like MM-PAMS tetramers, di-radical and saturated (Figures 1(a) and 1(c)), are used to study degradation processes. Previous research has confirmed the rationality of the structure selection ${ }^{[15,16]}$. The PESs of possible degradation paths for these structures are shown in Figures 1(b) and 1(d). For the di-radical structure, the energy barriers required for dissociation of the $\mathrm{C}$-unsaturated end and the $\mathrm{CH}_{2}$-unsaturated end to produce the monomer are R1 $(0.68 \mathrm{eV})$ and $\mathrm{R} 3(0.82 \mathrm{eV})$. This indicates that depolymerization occurs readily at the C-unsaturated end. In addition, the $\mathrm{H}$ atom transfer to both ends of the polymer chain (R2 and R5) will lead to the chain scission, which involves two steps. Taking R2 as an example, owing to the steric hindrance of $\mathrm{CH}_{2}$ in the ortho position, the $\mathrm{C}$ atom at the $\mathrm{C}$-unsaturated end will seize the $\mathrm{H}$ atom on the methyl group of the second monomer to produce an intermediate. Then, the chain scission occurs on two $\mathrm{C}-\mathrm{C}$ bonds adjacent to the $\mathrm{C}-\mathrm{C}$ bond where the methyl group on the second monomer is located. Thus there are two possible scission paths (R2-1/R2-2). In the first path, a monomer free radical and a chain with a $\mathrm{CH}_{2}$-unsaturated end are produced. In the second path, a dimer and an unsaturated chain form together. The reaction of R5 is similar to that of R2, and there are also two scission paths (R5-1/R5-2) afterwards. The difference is that there is a prerequisite for the occurrence of R5: the polymer must first undergo an isomerization process ( $\mathrm{R} 4)$.

Next, we analysed these multi-step reactions from the perspective of energy. The energy barrier of $\mathrm{R} 2(1.39 \mathrm{eV})$ is higher than that of R1 $(0.68 \mathrm{eV})$ and R3 $(0.82 \mathrm{eV})$, indicating that a depolymerization reaction is more likely to occur than a $\mathrm{H}$ transfer reaction at the $\mathrm{C}$-unsaturated end. Meanwhile, the energy barriers of R4 $(0.26 \mathrm{eV})$ and R5 $(0.60 \mathrm{eV})$ are lower than those of R1-R3, which suggests that the $\mathrm{CH}_{2}$ unsaturated end tends to cause isomerization rearrangement and then seizure of the $\mathrm{H}$ atom rather than depolymerization. In addition, the energy barriers of the two chain scission paths after R2 and R5 are 0.73/0.78 eV and 0.76/0.72 eV. These values are between those of R1 and R3, meaning that the chain scission reaction readily occurs after $\mathrm{H}$ transfer. In other words, the $\mathrm{H}$ transfer reaction contributes to the occurrence of chain scission reactions. In comparison, we also studied the degradation of saturated MM-PAMS. The transfer of $\mathrm{H}$ at both ends of the main chain causes the dissociation of the AMS monomer (R6/R7). As expected, their energy barriers $(3.57 / 4.23 \mathrm{eV})$ are much higher than those of other reactions.

Meanwhile, the selected frontier molecular orbitals (MOs) of the reactants in the R1-R4 and R6-R7 reactions are also given in Figure 1(e). The di-radical MM-PAMS possesses an electronic ground state of spin-polarized singlet state with antiferromagnetic coupling, in which net spin electrons are distributed at the two unsaturated ends. Obviously, the frontier MOs of the reactant in R1-R4 are localized at the two ends near the monomer linkage sites. Among them, HOMO- $\beta$ (highest occupied molecular orbital) and LUMO$\alpha$ (lowest unoccupied molecular orbital) are located at the 

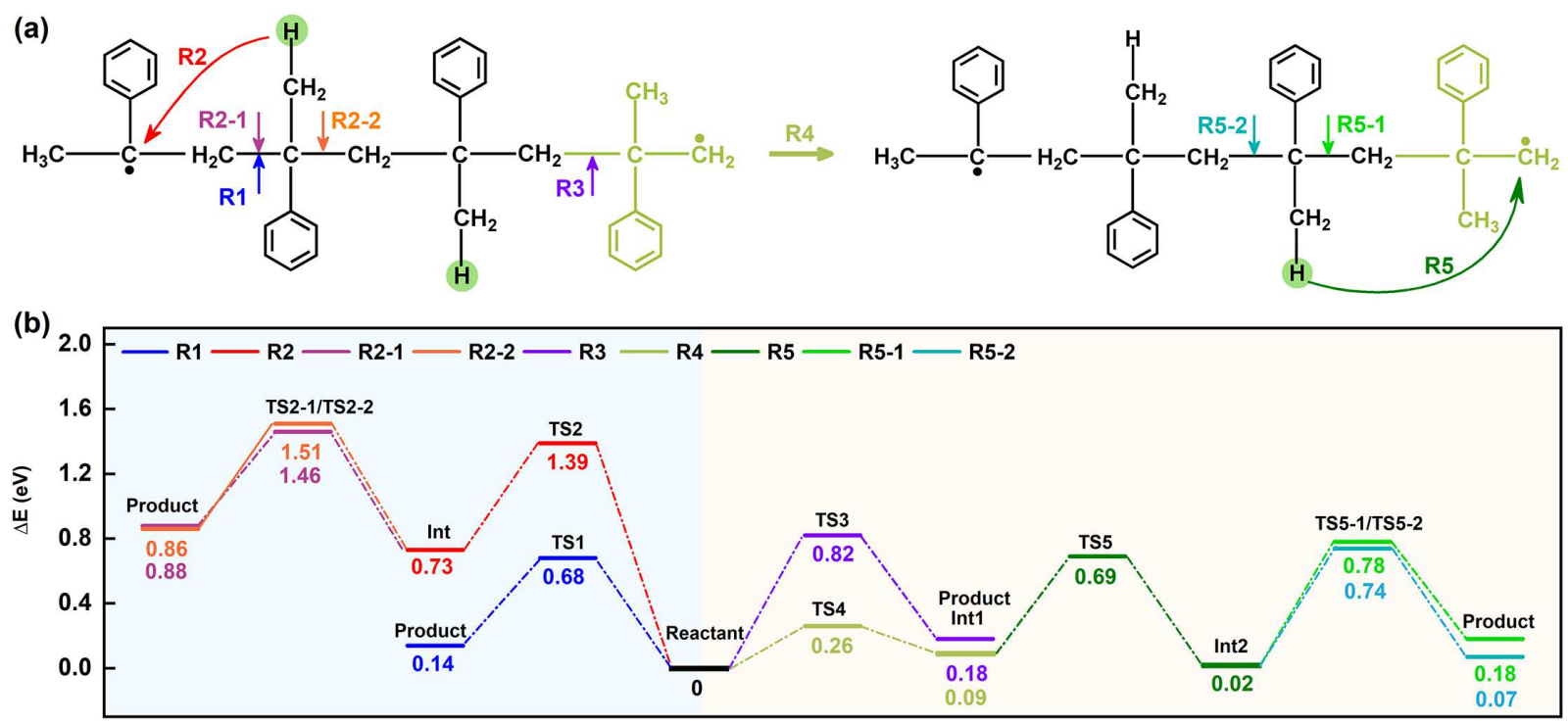

(c)
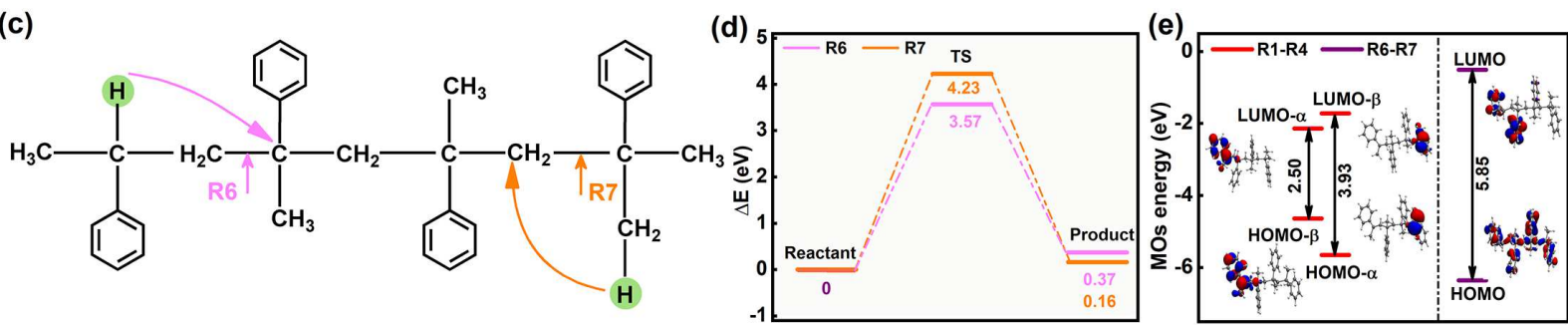

Figure 1. The degradation reactions of MM-PAMS. (a) Geometric structures of di-radical MM-PAMS. R1 and R3 are degradation paths that produce the $\alpha$ methylstyrene (AMS) monomer. R2 indicates the $\mathrm{H}$ atom transfer reactions. $\mathrm{R} 4$ is the isomerization process. $\mathrm{R} 5$ represents the $\mathrm{H}$ atom transfer reactions after R4. R2-1/R2-2 and R5-1/R5-2 are chain scission reactions after the corresponding $\mathrm{H}$ transfer reaction. The locations of arrows denote the bonds breaking on the corresponding degradation path. (b) Potential energy curves of these reactions for di-radical structure. 'Int' represents the intermediate. The energy of the reactant is taken as the zero. All energies were corrected with ZPE. The light blue and beige areas in the figure represent the degradation reactions related to the $\mathrm{C}$-unsaturated and $\mathrm{CH}_{2}$-unsaturated ends, respectively. (c) Geometric structures of saturated MM-PAMS. R6 and R7 are degradation reactions that produce the AMS monomer after $\mathrm{H}$ transfer of two ends. (d) Potential energy curves of two reactions for saturated structure. (e) Selected frontier molecular orbitals (MOs) of reactants in R1-R4 and R6-R7 reactions. Isovalue equals 0.035.

C-unsaturated end, and HOMO- $\alpha$ and LUMO- $\beta$ are located at the $\mathrm{CH}_{2}$-unsaturated end, indicating that these two positions have strong chemical reactivity and are prone to break. For the reactant in the R6/R7 reaction, its frontier MOs are distributed throughout the polymer chain instead of being localized at a single position. To a certain extent, this also explains why R1, R3 and R4, which involve a $\mathrm{C}-\mathrm{C}$ bond located at the monomer linkage site, occur more readily than other reactions. Moreover, the HOMO-LUMO gap of the reactant in R6/R7 $(5.85 \mathrm{eV})$ is much larger than that of the reactant in R1-R4 $(2.50 / 3.93 \mathrm{eV})$. This result further demonstrates that R6/R7 is the most difficult to occur. Thus, the differences in energy barriers and frontier MOs of these reactions suggest that depolymerization may be a fast process, and that most of the hydrogen-transfer-induced chain scission, except that which occurs at the $\mathrm{CH}_{2}$-unsaturated end, may be a slow process.

The geometric images before and after reactions are shown in Figure 2. In the degradation reactions (R1, R3) that produce the monomer, the reactants all broke the $\mathrm{C}-\mathrm{C}$ bond in the linkage site of the monomer. For the degradation
(R6, R7) of the saturated structures, the reactants not only broke the $\mathrm{C}-\mathrm{C}$ bond, but also the $\mathrm{C}-\mathrm{H}$ bond at the chain end. For the isomerization reaction (R4), compared with the reactant, the main change of product is the flip of the benzene ring at the $\mathrm{CH}_{2}$-unsaturated end. In the $\mathrm{H}$ transfer reactions (R2, R5), the reactants underwent the breaking and recombination process of the $\mathrm{C}-\mathrm{H}$ bond (the $\mathrm{H}$ atom on the methyl of the second/third monomer transfers to the $\mathrm{C}$ atom at the chain end). For chain scission reactions (R2-1, R2-2, R5-1, R5-2), the reactants all broke the C-C bonds adjacent to the $\mathrm{C}-\mathrm{C}$ bond where the methyl group on the second/third monomer is located. Therefore, for depolymerization and chain scission reactions, the change in geometric structure is mainly the breaking of the $\mathrm{C}-\mathrm{C}$ bond, whereas for $\mathrm{H}$ transfer reactions, it is the regeneration of the $\mathrm{C}-\mathrm{H}$ bond.

In order to verify the possibility of these reaction paths, we conducted the dynamic simulation on the evolution of the spatial structure over time based on the density functionalbased tight binding (DFTB) method. For the systems in this study, we chose the Slater-Koster-type parameters ${ }^{[30]}$ to 

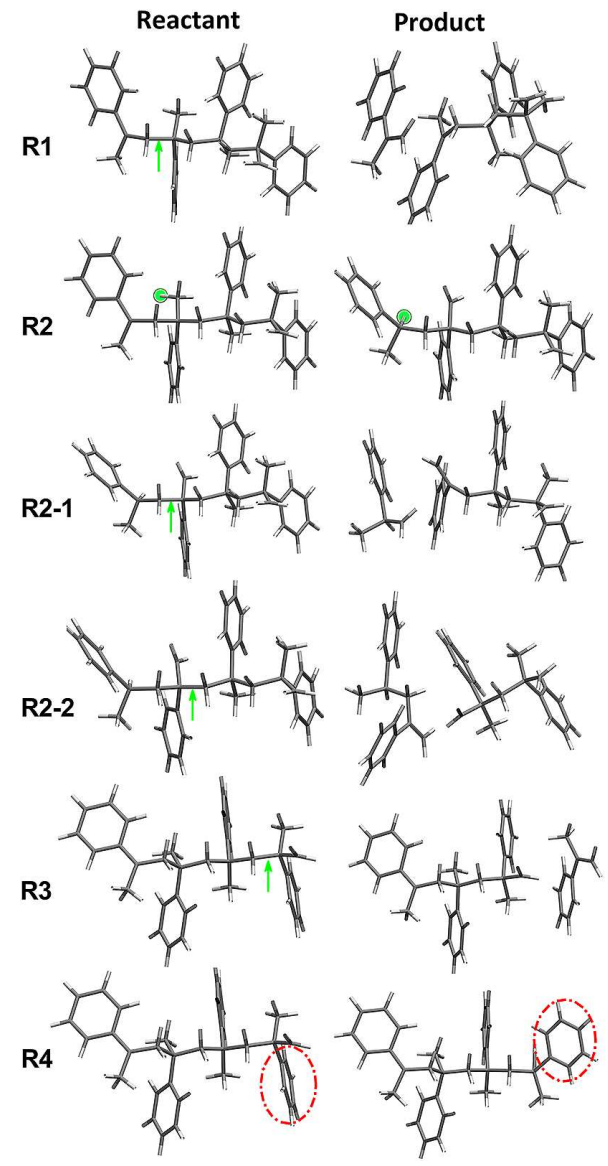
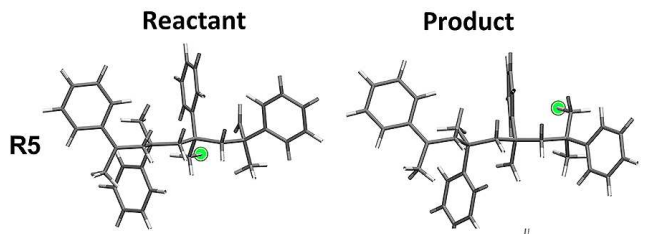

(1)
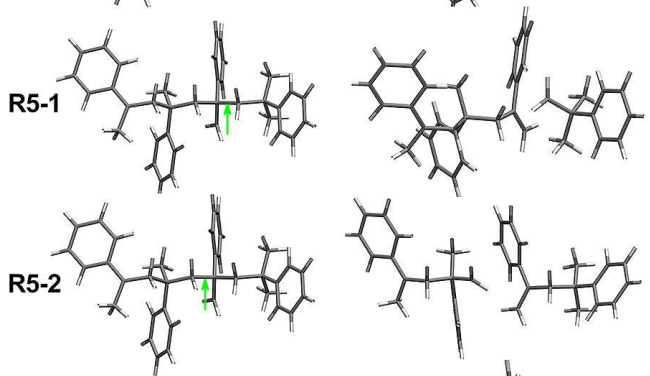

R6
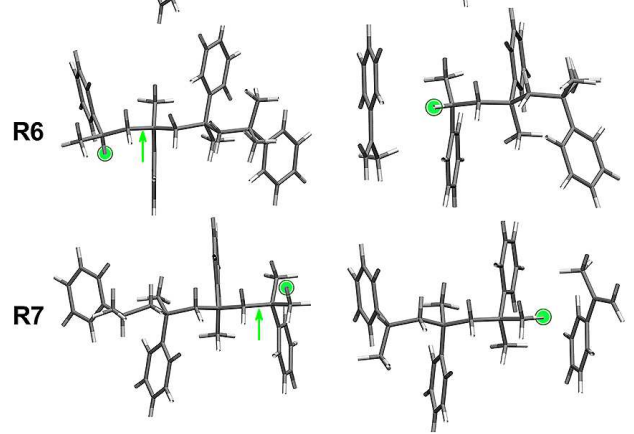

Note:

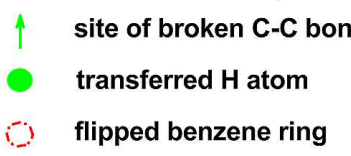

Figure 2. Geometric images before and after reactions. Here, the green arrows indicate the site of broken $\mathrm{C}-\mathrm{C}$ bonds, and the green circles and red dash circles, respectively, denote the $\mathrm{H}$ atom and benzene ring involved in reactions.

describe the connections between atoms. The DFTB method based on these parameters describes the geometric structure of a system containing elements such as $\mathrm{C}, \mathrm{H}, \mathrm{O}$ and $\mathrm{N}$ that is consistent with that described by the traditional DFT method $^{[32]}$. The changes in bond length over time at the position of the broken bond with a temperature of $550 \mathrm{~K}$ are shown in Figure 3. We found that di-radical MM-PAMS dissociates two monomers in sequence from the $\mathrm{C}$-unsaturated end (Figure 3(a)), which is generally known as the depolymerization reaction. Meanwhile, a $\mathrm{H}$ atom transfer reaction like R5 was also observed (Figure 3(b)). The C-unsaturated end of MM-PAMS first dissociates a monomer. Then, under the influence of dynamic processes, violent thermal vibration of the polymer chain leads to the 'back biting' of the $\mathrm{CH}_{2}$ unsaturated end. At this time, the primary $\mathrm{H}$ atom on the third monomer gradually transfers to the $\mathrm{C}$ atom (marked as $\mathrm{C} 4$ in Figure 3(b)) at the $\mathrm{CH}_{2}$-unsaturated end until it is completely seized by $\mathrm{C} 4$. Because the intermediate with a stable 6-membered ring can be produced during the $\mathrm{H}$ transfer process, this reaction is likely to occur. For saturated MMPAMS, no degradation was observed in any of the dynamic simulations. This suggests that a saturated polymer is difficult to degrade. These results are consistent with the law of PESs.
To further understand these degradation paths, their reaction rates were obtained by TS theory with Eckart tunnelling correction (TST/Eckart) ${ }^{[33,34]}$. The equation presented for TST/Eckart is

$$
v^{\mathrm{TST} / \mathrm{T}}(T)=\chi(T) \times v^{\mathrm{TST}}(T),
$$

where

$$
v^{\mathrm{TST}}(T)=\sigma \frac{k_{b} T}{h}\left(\frac{R T}{P^{0}}\right)^{\Delta n} \mathrm{e}^{\frac{-\Delta G^{\neq \neq, 0}(T)}{k_{b} T}}
$$

and $\chi(T)$ is the transmission coefficient. Considering that $\mathrm{H}$ transfer contributes to the occurrence of chain scission in multi-step reactions and the thermal stability temperature of GDP coating is usually no higher than $600 \mathrm{~K}$, we mainly focused on the reaction rates of $\mathrm{H}$ transfer and depolymerization in a temperature range of 300-600 K. Figures 4(a) and 4(b) show the rate constants of these reactions as a function of temperature. For the same temperature, the rates for the depolymerization reaction at the C-unsaturated end (R1) and the $\mathrm{CH}_{2}$-unsaturated end (R3), as well as the $\mathrm{H}$ transfer reaction at the $\mathrm{CH}_{2}$-unsaturated end (R5), are much greater than those of other reactions. Taking the temperature $550 \mathrm{~K}$ as an example, the reaction rates of R1, R3 and R5 
are between $2.86 \times 10^{5} \mathrm{~s}^{-1}$ and $4.13 \times 10^{7} \mathrm{~s}^{-1}$, whereas the reaction rates of other reactions are between $6.90 \times 10^{-25}$ and $1.67 \mathrm{~s}^{-1}$. Surprisingly, the reaction rates of R1, R3 and R5 are 5-31 orders of magnitude faster than those of other reactions. This demonstrates that these reactions are fast processes, whereas other reactions are slow processes during degradation, which agrees with the comparison result of energy barriers (Figures 1(b) and 1(d)). Thus, a degradation reaction model that combines fast and slow processes is established at the atomic level.

In addition, the reaction rate of slow processes (R2, R6 and R7) is greatly affected by temperature. Particularly for chain scission reactions at the $\mathrm{C}$-end (R6) and $\mathrm{CH}_{2}$-end
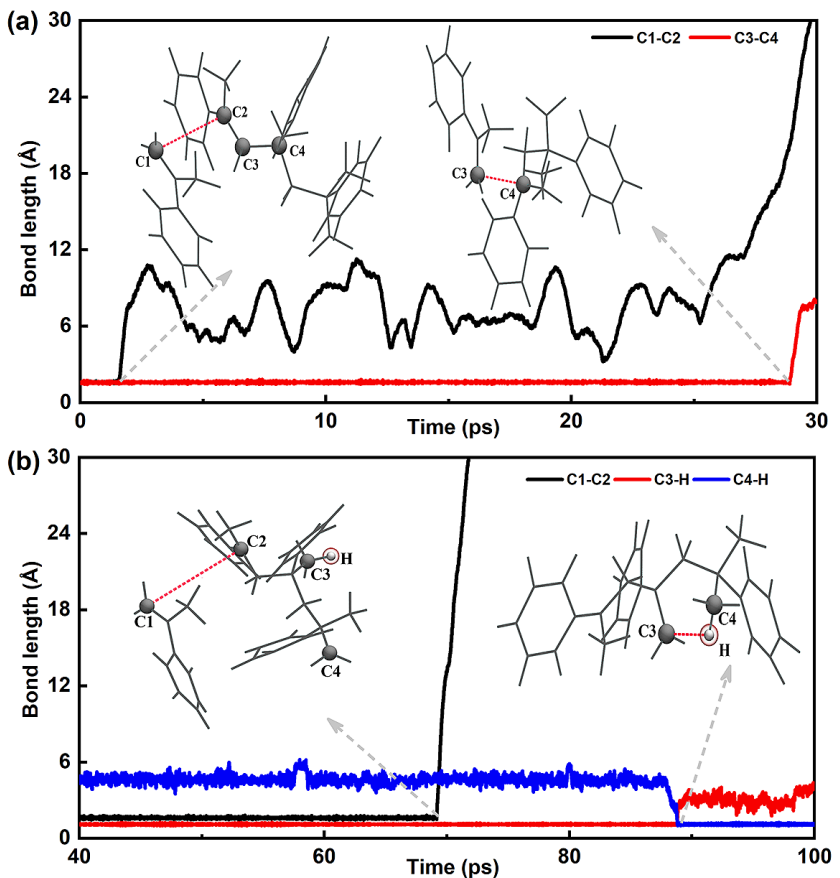

Figure 3. Dynamic simulations of the degradation processes of di-radical MM-PAMS at $550 \mathrm{~K}$. These figures represent the bond length changes with respect to time for breaking sites on the backbone chain. Typical conformations during degradation are also drawn. C1-C2, C3-C4 and so on are the positions of broken bonds during degradation.
(R7) of saturated MM-PAMS, as the temperature increases, a significant magnitude change in the reaction rate can be observed. It suggests that the degradation reaction is determined by slow processes, which require more complex experiment control. Moreover, for R1 and R5, we noticed alternate high and low changes in the reaction rate dependent on temperature. In order to visually see the change, the ratios of two reaction rates to the total rate were calculated, as shown in Figure 4(c). When the temperature is lower than $472 \mathrm{~K}, \mathrm{R} 5$ is the main contributor to degradation. As the temperature increases, R1 gradually dominates. This phenomenon explains the fact that the monomer yield is extremely high when the temperature is over $550 \mathrm{~K}$ in the experiment ${ }^{[14]}$, which provides a reference for the suitable degradation temperature of MM-PAMS.

We then performed the thermal degradation kinetics analysis using the TG curve measured by experiment and the Arrhenius law. In our previous work, the TG curve of MMPAMS was obtained ${ }^{[17]}$. In this study, in order to compare the weight loss rate at different temperatures more clearly, we calculated the DTG curve (Figure 5(a)). There are three peaks in the DTG curve, which correspond to the temperatures of $427 \mathrm{~K}, 520 \mathrm{~K}$ and $571 \mathrm{~K}$. The weight loss rate at these three temperatures gradually increased, reaching the maximum at about $571 \mathrm{~K}$. This can be explained by the reaction rates of the depolymerization reaction at the $\mathrm{C}$ unsaturated end (R1) and the $\mathrm{H}$ transfer reaction at the $\mathrm{CH}_{2}$ unsaturated end (R5), as shown in Figure 4(c). R5 is the main contributor to the degradation reaction below $472 \mathrm{~K}$, so the weight loss is small at $427 \mathrm{~K}$. After that, as the temperature increases, R1 gradually dominates, leading to a significant increase in weight loss. These results once again prove that MM-PAMS degradation is a complex process, with many other degradation paths apart from depolymerization.

Furthermore, from the TG data obtained from experiment, we selected data in the temperature range (528-583 K) containing the main peak, to analyse the thermal degradation kinetics. For thermal degradation of MM-PAMS, according to the Arrhenius equation $k=A \mathrm{e}^{\frac{-E}{R T}}$ (where $A$ is the frequency
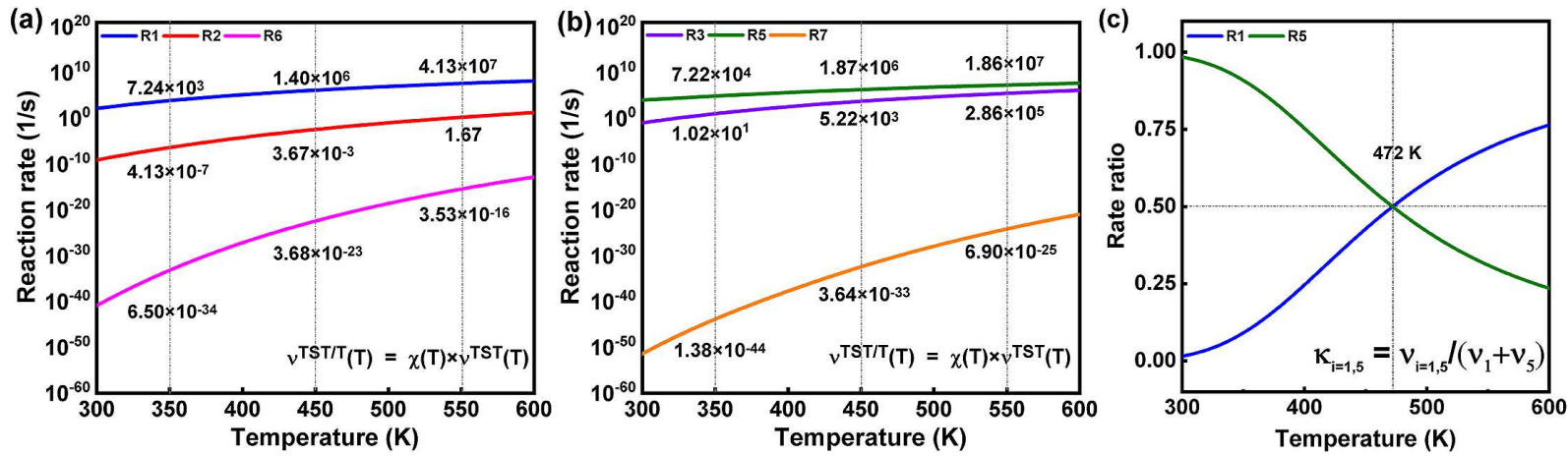

Figure 4. Reaction rate constants and ratios of degradation reactions as functions of temperature. (a) Reaction rates of degradation reactions related to the C-unsaturated end. (b) Reaction rates of degradation reactions related to the $\mathrm{CH}_{2}$-unsaturated end. All values were calculated by TST/Eckart using the KiSThelP program ${ }^{[33]}$. In both (a) and (b), values of these reaction rates corresponding to $350 \mathrm{~K}, 450 \mathrm{~K}$ and $550 \mathrm{~K}$ are also marked. (c) Reaction rate ratios of R1 and R5. The equations in the lower right corner of each graph represent the calculation formulas of rate constant and ratio, respectively. 
(a)

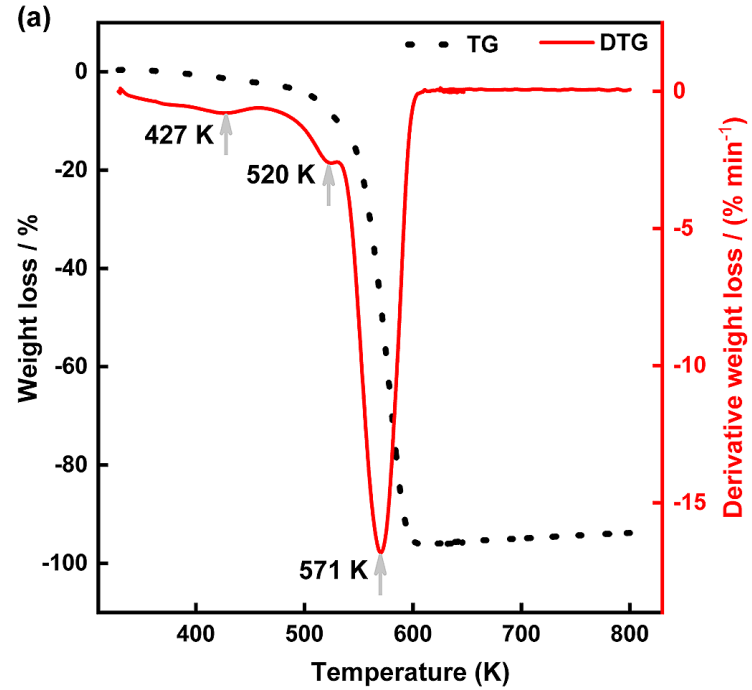

(b)

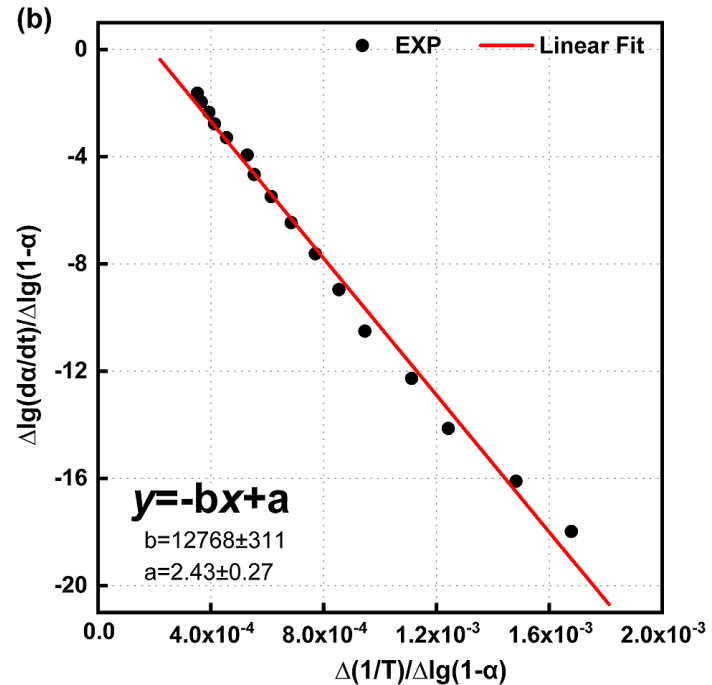

Figure 5. Degradation experiment on MM-PAMS. (a) The TG and derivative thermogravimetric (DTG) curves of MM-PAMS. The black dotted line is the TG curve, which has been shown in our previous work ${ }^{[17]}$. The red line represents the DTG curve. The grey arrows represent the locations of three peaks in the DTG curve. (b) The thermal degradation kinetics of MM-PAMS. Black dots represent values obtained through experiment. The solid line is the fit for the function $\frac{\Delta \lg (\mathrm{d} \alpha / \mathrm{d} t)}{\Delta \lg (1-\alpha)}=-\frac{E}{2.33 R} \times \frac{\Delta(1 / T)}{\Delta \lg (1-\alpha)}+n$ (see the text for a detailed description).

factor, $E$ is the activation energy and $R$ is the gas constant), the reaction rate can be expressed as

$$
\frac{\mathrm{d} \alpha}{\mathrm{d} t}=A \mathrm{e}^{\frac{-E}{R T}}(1-\alpha)^{n}
$$

where $\alpha=\frac{m_{0}-m_{t}}{m_{0}}$ represents the mass change rate, $m_{0}$ and $m_{t}$ are the initial mass and the mass at time $t$, respectively; $k(\alpha)=\frac{\mathrm{d} \alpha}{\mathrm{d} t}$ indicates the decomposition rate and $(1-\alpha)^{n}$ is the function of thermal weight-loss rate. By taking the logarithm and differentiating on both sides of Equation (3), it can be simplified as follows:

$$
\frac{\Delta \lg (\mathrm{d} \alpha / \mathrm{d} t)}{\Delta \lg (1-\alpha)}=-\frac{E}{2.33 R} \times \frac{\Delta(1 / T)}{\Delta \lg (1-\alpha)}+n .
$$

Using the TG data, we calculated relevant variables $(\alpha, 1-$ $\alpha, \mathrm{d} \alpha / \mathrm{d} t, \lg (1-\alpha), 1 / T)$ and substituted them into Equation (4). Thus, the relationship between $\frac{\Delta \lg (\mathrm{d} \alpha / \mathrm{d} t)}{\Delta \lg (1-\alpha)}$ and $\frac{\Delta(1 / T)}{\Delta \lg (1-\alpha)}$ was obtained, as shown in Figure 5(b). The two expressions display a linear relationship. From the slope of the line, the activation energy $E=2.53 \mathrm{eV}$ was calculated. Compared with the energy barriers (Figures 1(b) and 1(d)) obtained by first-principles calculation, it can be seen that this value of $2.53 \mathrm{eV}$ is between the energy barriers of fast and slow processes, indicating that the activation energy is not associated with a separate depolymerization or hydrogen-transferinduced chain scission, but is the average value of multiple reaction paths. To verify this, we analysed the energy barriers of fast and slow processes and found that the value was slightly larger than the average energy barrier $(2.48 \mathrm{eV})$ of two slow processes (R2, R6). This result further highlights the fact that slow processes play a decisive role in MMPAMS degradation.

\section{Conclusion}

In summary, by studying the degradation processes of diradical and saturated MM-PAMS, we proposed a mandrel degradation model combining with fast and slow processes at the atomic level. The calculations of PESs show that there are two degradation paths at both ends of the main chain: depolymerization and hydrogen-transfer-induced chain scission reaction. Between them, the energy barrier for the former $(0.68-0.82 \mathrm{eV})$ is smaller than that for most of the latter $(1.39-4.23 \mathrm{eV})$. In particular, when the latter occurs at the $\mathrm{CH}_{2}$-unsaturated end, its energy barrier can be even lower than that of depolymerization. More importantly, the calculated reaction rates indicate orders of magnitude differences in reaction rates for depolymerization and most of the hydrogen-transfer-induced chain scission differs greatly at the same temperature. At $550 \mathrm{~K}$, the difference can reach 5-31 orders of magnitude. Thus, it is evident that depolymerization is a fast process and hydrogen-transfer-induced chain scission, except that at the $\mathrm{CH}_{2}$-unsaturated end, is a slow process. Not only that, this also clearly highlights the fact that the degradation reaction is determined by slow processes. Actually, slow processes lead to the generation of degradation sites, thereby promoting subsequent degradation, and fast processes quickly remove monomers one by one in the form of a zipper. Furthermore, TG experiments also confirmed the complexity of degradation and the importance of slow processes. We hope these findings provide new insight for 
relevant regulation and contribute to achieving more efficient degradation of mandrel for fabrication of ICF targets.

\section{Acknowledgements}

This work was supported by the National Natural Science Foundation of China (Nos. 11974136 and 11674123). Z. W. also acknowledges the High-Performance Computing Center of Jilin University.

\section{References}

1. E. M. Fearon, S. A. Letts, L. M. Allison, and R. C. Cook, Fusion Technol. 31, 406 (1997).

2. B. W. McQuillan, A. Nikroo, D. A. Steinman, F. H. Elsner, D. G. Czechowicz, M. L. Hoppe, M. Sixtus, and W. J. Miller, Fusion Technol. 31, 381 (1997).

3. A. Nikroo and J. M. Pontelandolfo, Fusion Technol. 38, 58 (2000).

4. S. A. Letts, E. Fearon, L. Allison, S. Buckley, M. Saculla, and R. Cook, "Decomposable mandrel project - progress report," Lawrence Livermore National Laboratory, UCRL-ID-120913 (1995).

5. H. H. G. Jellinek and H. Kachi, J. Polym. Sci. Part C: Polym. Symp. 23, 97 (1968).

6. G. Madras, J. M. Smith, and B. J. McCoy, Polym. Degrad. Stab. 52, 349 (1996).

7. M. Hoppe, Fusion Technol. 38, 42 (2000).

8. A. B. Hersberger, J. C. Reid, and R. G. Heiligmann, Ind. Eng. Chem. Res. 37, 1073 (1945).

9. R. Simha, L. A. Wall, and P. J. Blatz, J. Polym. Sci. 5, 615 (1950).

10. D. W. Brown and L. A. Wall, J. Phys. Chem. 62, 848 (1958).

11. S. L. Malhotra, C. Baillet, L. Minh, and L. P. Blanchard, J. Macromol. Sci. Part A: Chem. 12, 129 (1978).

12. G. Madras, G. Y. Chung, J. M. Smith, and B. J. McCoy, Ind. Eng. Chem. Res. 36, 2019 (1997).

13. W. J. Sterling, Y.-C. Kim, and B. J. McCoy, Ind. Eng. Chem. Res. 40, 1811 (2001).

14. M. Chen, Z. Zhang, Y. Yi, H. Wang, Y. Huang, and R. Shi, Polym. Mater. Sci. Eng. 34, 172 (2018).

15. T. Yu, Y. Gao, B. Wang, X. Dai, W. Jiang, R. Song, Z. Zhang, M. Jin, Y. Tang, and Z. Wang, Chem. Phys. Chem. 16, 3308 (2015).

16. Y. Zhu, R. Wang, W. Xie, D. Zhang, Z. Zhang, and Z. Wang, Phys. Chem. Chem. Phys. 21, 16905 (2019).

17. Z. W. Zhang, Y. Huang, X. Luo, X. B. Qi, S. F. Chen, Y. Y. Liu, and B. Li, High Power Laser Part. Beams 22, 2079 (2010).

18. A. D. Becke, J. Chem. Phys. 140, 18A301 (2014).

19. R. O. Jones, Rev. Mod. Phys. 87, 897 (2015).
20. M. R. Siebert, J. M. Osbourn, K. M. Brummond, and D. J. Tantillo, J. Am. Chem. Soc. 132, 11952 (2010).

21. C. Tian, P. Xiu, Y. Meng, W. Zhao, Z. Wang, and R. Zhou, Chem. Eur. J. 18, 14305 (2012).

22. C. Lee, W. Yang, and R. G. Parr, Phys. Rev. B 37, 785 (1988).

23. A. D. Becke, J. Chem. Phys. 98, 5648 (1993).

24. K. Fukui, J. Phys. Chem. 74, 4161 (1970).

25. C. Gonzalez and H. B. Schlegel, J. Phys. Chem. 94, 5523 (1990).

26. M. J. Frisch, G. W. Trucks, H. B. Schlegel, G. E. Scuseria, M. A. Robb, J. R. Cheeseman, G. Scalmani, V. Barone, B. Mennucci, G. A. Petersson, H. Nakatsuji, M. Caricato, X. Li, H. P. Hratchian, A. F. Izmaylov, J. Bloino, G. Zheng, J. L. Sonnenberg, M. Hada, M. Ehara, K. Toyota, R. Fukuda, J. Hasegawa, M. Ishida, T. Nakajima, Y. Honda, O. Kitao, H. Nakai, T. Vreven, J. A. Montgomery, Jr., J. E. Peralta, F. Ogliaro, M. Bearpark, J. J. Heyd, E. Brothers, K. N. Kudin, V. N. Staroverov, R. Kobayashi, J. Normand, K. Raghavachari, A. Rendell, J. C. Burant, S. S. Iyengar, J. Tomasi, M. Cossi, N. Rega, J. M. Millam, M. Klene, J. E. Knox, J. B. Cross, V. Bakken, C. Adamo, J. Jaramillo, R. Gomperts, R. E. Stratmann, O. Yazyev, A. J. Austin, R. Cammi, C. Pomelli, J. W. Ochterski, R. L. Martin, K. Morokuma, V. G. Zakrzewski, G. A. Voth, P. Salvador, J. J. Dannenberg, S. Dapprich, A. D. Daniels, Ö. Farkas, J. B. Foresman, J. V. Ortiz, J. Cioslowski, and D. J. Fox, Gaussian 09, Revision D.01 (Gaussian, Inc., Wallingford, 2009).

27. Y. Kitagawa, T. Saito, M. Ito, M. Shoji, K. Koizumi, S. Yamanaka, T. Kawakami, M. Okumura, and K. Yamaguchi, Chem. Phys. Lett. 442, 445 (2007).

28. T. Saito, S. Nishihara, Y. Kataoka, Y. Nakanishi, T. Matsui, Y. Kitagawa, T. Kawakami, M. Okumura, and K. Yamaguchi, Chem. Phys. Lett. 483, 168 (2009).

29. M. Elstner, T. Frauenheim, and S. Suhai, J. Mol. Struc: Theochem 632, 29 (2003).

30. M. Elstner, D. Porezag, G. Jungnickel, J. Elsner, M. Haugk, T. Frauenheim, S. Suhai, and G. Seifert, Phys. Rev. B 58, 7260 (1998).

31. B. Hourahine, B. Aradi, V. Blum, F. Bonafé, A. Buccheri, C. Camacho, C. Cevallos, M. Y. Deshaye, T. Dumitrică, A. Dominguez, S. Ehlert, M. Elstner, T. van der Heide, J. Hermann, S. Irle, J. J. Kranz, C. Köhler, T. Kowalczyk, T. Kubař, I. S. Lee, V. Lutsker, R. J. Maurer, S. K. Min, I. Mitchell, C. Negre, T. A. Niehaus, A. M. N. Niklasson, A. J. Page, A. Pecchia, G. Penazzi, M. P. Persson, J. Xezč, C. G. Sánchez, M. Sternberg, M. Stöhr, F. Stuckenberg, A. Tkatchenko, V. M. Z. Yu, and T. Frauenheim, J. Chem. Phys. 152, 124101 (2020).

32. T. Krüger, M. Elstner, P. Schiffels, and T. Frauenheim, J. Chem. Phys. 122, 114110 (2005).

33. S. Canneaux, F. Bohr, and E. Henon, J. Comput. Chem. 35, 82 (2013).

34. C. Eckart, Phys. Rev. 35, 1303 (1930). 